\title{
Calculation of the Angular Radiance Distribution for a Coupled Atmosphere and Canopy
}

\author{
Shunlin Liang and Alan H. Strahler, Member, IEEE
}

\begin{abstract}
The radiative transfer equations for a coupled atmosphere and canopy are solved numerically by an improved Gauss-Seidel iteration algorithm. The radiation field is decomposed into three components: unscattered sunlight, single scattering, and multiple scattering radiance for which the corresponding equations and boundary conditions are set up and their analytical or iterational solutions are explicitly derived. The classic Gauss-Seidel algorithm has been widely applied in atmospheric research. This is its first application for calculating the multiple scattering radiance of a coupled atmosphere and canopy. This algorithm enables us to obtain the internal radiation field as well as radiances at boundaries. Any form of bidirectional reflectance distribution function (BRDF) as a boundary condition can be easily incorporated into the iteration procedure. The hotspot effect of the canopy is accommodated by means of the modification of the extinction coefficients of upward single scattering radiation and unscattered sunlight using the formulation of Nilson and Kuusk. To reduce the computation for the case of large optical thickness, an improved iteration formula is derived to speed convergence. The upwelling radiances have been evaluated for different atmospheric conditions, leaf area index (LAI), leaf angle distribution (IAD), leaf size and so on. The formulation presented in this paper is also well suited to analyze the relative magnitude of multiple scattering radiance and single scattering radiance in both the visible and near infrared regions.
\end{abstract}

Keywords - Radiative transfer, bidirectional reflectance distribution function (BRDF), leaf canopy, atmosphere, radiance.

\section{INTRODUCTION}

$\mathbf{C}$ ONVENTIONAL uses of remote sensing have emphasize near-nadir reflectance measurements to identify terrestrial objects and determine their characteristics. Unfortunately, nadir reflectances are inadequate for many purposes because surface covers often have the same or nearly the same nadir reflectance patterns and because natural terrestrial surfaces reflect radiation anisotropically. The limitations of nadir reflectances in classification and other analyses have led to alternative approaches in the use of multiangle imagery.

Several s€nsors with multiple viewing angles, proposed as part of the liarth Observation System (EOS), will offer new capabilities for remotely observing directional variations in surface reflectance. In the pre-EOS era, there is at least one aircraft mounted sensor that can acquire off-nadir imagery - the Advanced Solid-state Array Spectroradiometer (ASAS) [1].

Manuscript received March 5, 1992; revised August 12, 1992. This work was supported by the National Aeronautics and Space Administration under Grant NAGW-2082, Contracts NAS5-30917 and NAS5-31369, and the U.S. National Science Foundation under Grant INT-9014263.

The authors are with the Department of Geography and the Center for Remote Sensing, Boston University, Boston, MA 02215.

IEEE Log N umber 9206930.
This has produced a number of directional datasets for research applications.

These developments provide unique opportunities for various scientific communities, but the potential utilization of directional data to retrieve quantitative estimates of land surface properties is highly dependent on our understanding of the physical processes, which is often inadequate. Development of physical models describing such processes is required.

The radiative transfer theory for a turbid medium has been widely employed for many years, but for vegetation canopies, the classic theory and its mathematical approaches are inappropriate because of the large size of canopy leaves and the important role played by the geometrical structure of the plant stand in its radiation regime. Some different modifications of the classic radiative transfer theory for canopies have been proposed in the past years [2]-[4]. The presence of oriented leaves makes the scattering by the plant medium much more complicated. Various forms of scattering phase functions based on rotationally invariant scattering models, such as Henyey-Greenstein functions, are not appropriate cor the leaf canopy problem. In this paper, a simple but realistic leaf scattering model called the bi-Lambertian scattering model is used. Proposed by Ross and Nilson [5], it has been widely applied [6], [7]. Marshak [8] extended it to incorporate a specular component of reflection and to consider the hotspot effect, and Myneni et al. [9] used this model within a threedimensional radiative transfer framework.

Most canopy reflectance models decouple atmospheric scattering and assume that the diffuse flux from the atmosphere into the canopy is isotropic. But the real distribution of sky radiance is not so simple [10], [11], and realistic models are required for the coupled atmosphere and canopy. In an earlier study, Weinman and Guetta [12] used the two-strcam approximation to describe fluxes for a coupled atmosphere and canopy. In their model, empirical coefficients for the canopy were employed. Gerstl and Zardecki [13] used the discrete-ordinates finite element method to study the radiance distribution. However, they assumed isotropic scattering in the canopy, which for realistic canopies can give poor approximations for the reflected angular flux [6]. In a later study [14], they used the Henyey-Greenstein phase function as an approximation to the actual scattering phase function of the canopy.

Many numerical approaches to obtain an accurate solution to the radiative transfer equation for canopy problems have been published, including successive order of scattering approximations (SOSA) [15], discrete ordinates [13], [16], [17], 


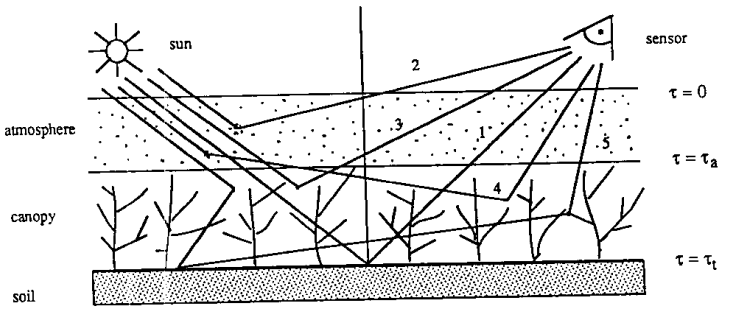

Fig. 1. Schematic illustration of radiation field on the coupled atmospherecanopy medium and its decomposition.

the doubling or adding method [18], the integral-equation method [7], and the recent simplified $X$ and $Y$ function method [19]. This paper will explore the Gauss-Seidel algorithm in application to the coupled atmosphere-canopy radiative transfer problem. This algorithm has been widely used in atmospheric radiative transfer work [20]-[22], but no application has been reported to solve the canopy radiative transfer equation, specifically for a coupled atmosphere and canopy. The technique employing a Gauss-Seidel iteration is attractive, as it does not require any more time or complexity to handle vertical inhomogeneities, absorption, emission, scattering, and any form of soil directional reflectance function. A corresponding algorithm suitable for supercomputer parallel implementation can be easily developed. The major limitation of the Gauss--Seidel algorithm for canopy problems is that it becomes too tedious for deep plant canopies. In this paper, an improved version is developed to speed up the convergence for the coupled system.

Often the solution to the radiative transfer equation is presented in terms of directional reflectance functions. However, our primary survey has found some confusion in the literature regarding the use of reflectance concepts for directional functions. In this paper, the absolute radiance will be used instead of a reflectance function. Also, the emphasis will be put on the angular characteristics of the radiation field of the coupled medium instead of wavelength dependence.

\section{DECOMPOSITION OF RADIATION FIELD}

Although we focus on the radiation field of the atmosphere and canopy as a single coupled medium, the radiative transfer models of atmosphere and canopy will be separately described because of their different attenuating properties. The coupled medium is illustrated in Fig.1, in which optical depth $\tau$ replaces geometric altitude $z$. The top of atmosphere is set to $\tau=0$, the bottom is set to $\tau_{a}$, and the total optical depth is $\tau_{t}$. Therefore the optical depth of the canopy is $\tau_{t}-\tau_{a}$, which may be interpreted as the leaf area index (LAI). (Table I defines the mathematical notation used in this text.) Both atmosphere and canopy are assumed horizontally infinite and homogeneous. Thus one-dimensional radiative transfer equations considering only vertical variations are dealt with in this paper. The general radiative transfer equation and definitions of related variables are given in an appendix.

In order to characterize the hotspot phenomenon effectively and obtain stable solutions of multiple scattering, the radiation field is decomposed into three parts; unscattered radiance
TABLE I

NOTATION

\begin{tabular}{|c|c|}
\hline Symbol & Explanation \\
\hline$\delta$ & Dirac delta function \\
\hline$F_{0}$ & extraterrestrial solar irradance \\
\hline$F(\cdot)$ & Fresnel reflectance function \\
\hline$f_{s}\left(\Omega^{\prime}, \Omega\right)$ & BRDF of soil \\
\hline$g_{1}(\Omega)$ & distribution function of the leaf normal orientation \\
\hline$\Gamma\left(\Omega^{\prime}, \Omega\right)$ & area scattering transfer function of canopy \\
\hline $\begin{array}{l}H \\
i_{0}\end{array}$ & $\begin{array}{l}\text { height of canopy in meters. } \\
\text { extraterrestrial solar net flux incident on the top of } \\
\text { atmosphere }\end{array}$ \\
\hline$I^{0}(\tau, \Omega)$ & unscattered solar radiance \\
\hline$I^{1}(\tau, \Omega)$ & single scattering radiance \\
\hline$I^{M}(\tau, \Omega)$ & multiple scattering radiance \\
\hline$J(\tau, \Omega)$ & source function of radiative transfer \\
\hline$k$ & leaf dimension parameter \\
\hline$\lambda$ & wavelength \\
\hline LAD & leaf angle distribution \\
\hline LAI & leaf area index \\
\hline$\mu_{0}$ & cosine of solar zenith angle \\
\hline$n$ & leaf wax refractive index \\
\hline$N$ & $\begin{array}{l}\text { total number of layers of the coupled medium split for } \\
\text { multiple scattering calculation, each of thickness } \Delta \tau \text {. }\end{array}$ \\
\hline$\Omega(\mu, \phi)$ & $\begin{array}{l}\text { solid angle consisting of cosine of zenith angle } \mu \text { and } \\
\text { azimuth angle } \phi\end{array}$ \\
\hline$P\left(\Omega^{\prime}, \Omega\right)$ & phase function of atmosphere \\
\hline$\phi_{0}$ & solar azimuth angle \\
\hline$r_{l}$ & leaf reflectance \\
\hline$R_{s}\left(\Omega^{\prime}, \Omega\right)$ & bidirectional reflectance factor of soil surface \\
\hline $\begin{array}{c}R_{s} \\
\tau\end{array}$ & $\begin{array}{l}\text { reflectance of Lambertian surface } \\
\text { optical depth of medium }\end{array}$ \\
\hline$\tau_{a}$ & $\begin{array}{l}\text { optical depth or meoum } \\
\text { atmospheric optical depth }\end{array}$ \\
\hline$\tau_{a e}$ & aerosol optical depth \\
\hline$\tau_{r}$ & molecular optical depth \\
\hline$\tau_{t}$ & $\begin{array}{l}\text { total optical depth of the coupled atmosphere-canopy } \\
\text { medium }\end{array}$ \\
\hline$t_{l}$ & leaf transmittance \\
\hline$u_{l}(z)$ & $\begin{array}{l}\text { leaf area density } \\
\text { single scattering albedo }\end{array}$ \\
\hline$\omega$ & single scattering albedo \\
\hline
\end{tabular}

(Only the main symbols used in the paper are listed. Transient notation and symbols generated by subscriptions and superscriptions are omitted here.)

$I^{0}(\tau, \Omega)$, single scattering radiance $I^{1}(\tau, \Omega)$, and multiple scattering radiance $I^{M}(\tau, \Omega)$

$$
I(\tau, \Omega)=I^{0}(\tau, \Omega)+I^{1}(\tau, \Omega)+I^{M}(\tau, \Omega) .
$$

A simple scheme is represented in Fig. 1. $I^{0}(\tau, \Omega)$ is denoted by 1 , which is neither scattered by the atmosphere nor canopy, but is reflected directly by the soil surface. $I^{1}(\tau, \Omega)$ is radiance either scattered once by the atmosphere, denoted by 2 , or once by the canopy, denoted by $3 . I^{M}(\tau, \Omega)$ is the most complicated component, which includes all of other components in the radiation field of the coupled medium. Two of them are illustrated by 4 and 5 in the figure.

Unscattered sunlight radiances $I^{0}(\tau, \Omega)$ are characterized by the following radiative transfer equation and corresponding boundary conditions. When $\tau<\tau_{a}$,

$$
\begin{cases}-\mu \frac{\partial I^{0}(\tau, \Omega)}{\partial \tau}+I^{0}(\tau, \Omega)=0 & \\ I^{0}(0, \Omega)=\delta\left(\Omega-\Omega_{0}\right) i_{0} & \mu<0 \\ I^{0}\left(\tau_{a}^{b o t}, \Omega\right)=I^{0}\left(\tau_{c}^{t o p}, \Omega\right) & \mu>0\end{cases}
$$


where $\tau_{a}^{b o t}$ and $\tau_{c}^{\text {top }}$ are the optical depths at the bottom of the atmosphere and the top of the canopy, respectively. Here different notations have been used to indicate the physical meaning of the boundary condition. The second equation in (2) gives the upper boundary condition, which means only parallel sunlight illuminates the atmosphere at the top in the direction $\Omega_{0}$. When $\tau>\tau_{a}$,

$$
\begin{cases}-\mu \frac{\partial^{0}(\tau, \Omega)}{\partial \tau}+h(\tau, \Omega) G(\Omega) I^{0}(\tau, \Omega)=0 & \\ I^{0}\left(\tau_{c}^{t \rho p}, \Omega\right)=I^{0}\left(\tau_{a}^{b o t}, \Omega\right) & \mu<0 \\ I^{0}\left(\tau_{t} \cdot \Omega=f_{s}\left(\Omega_{0}, \Omega\right)\left|\mu_{0}\right| I^{0}\left(\tau_{t}, \Omega_{0}\right)\right. & \mu>0 .\end{cases}
$$

Jointly solving (2) and (3) with these boundary conditions, it is easy to obtain

$$
\begin{aligned}
& I^{0}(\tau, \Omega)= \\
& \begin{cases}I_{d 1}^{0}(\tau, \Omega)=i_{0} \exp (-\tau /|\mu|) \delta\left(\Omega-\Omega_{0}\right) & \mu<0, \tau \leq \tau_{a} \\
I_{d 2}^{0}(\tau, \Omega)=I_{d 1}^{0}\left(\tau_{a}, \Omega\right) & \mu<0, \tau_{a}<\tau \leq \tau_{t} \\
\cdot \exp \left[-C(\Omega)\left(\tau-\tau_{a}\right) /|\mu|\right] & \\
I_{u 2}^{0}(\tau, \Omega)=: i_{0} & \\
\cdot \exp \left[-\frac{\tau_{a}+\left(\tau_{t}-\tau_{a}\right) G\left(\Omega_{0}\right)}{\left|\mu_{0}\right|}\right] & \mu>0, \tau_{a}<\tau \leq \tau_{t} \\
\cdot f_{s}\left(\Omega_{0}, \Omega\right)\left|\mu_{0}\right| \exp [-\xi(\tau, \Omega)] & \mu>0, \tau \leq \tau_{a} \\
I_{u 1}^{0}(\tau, \Omega)=I_{u 2}^{0}\left(\tau_{a}, \Omega\right) & \\
\cdot \exp \left[-\left(\tau_{a}-\tau\right) / \mu\right] & \end{cases}
\end{aligned}
$$

where $I_{u 2}^{0}(\tau, \Omega)$ represents the upwelling sunlight radiance within the canopy, and the function $\xi(\tau, \Omega)$, due to modifying the extinction coefficient of the canopy, has been evaluated by Marshak [8]

$$
\begin{aligned}
\xi(\tau, \Omega) & =\frac{1}{\mu} \int_{\tau}^{\tau_{t}} h(t, \Omega) G(\Omega) d t \\
& =G(\Omega) \frac{\tau_{t}-\tau}{\mu}-\left[\sqrt{\frac{G\left(\Omega_{0}\right) G(\Omega)}{\mu\left|\mu_{0}\right|}} \frac{k H}{\Delta\left(\Omega_{0}, \Omega\right)}\right] t_{0}
\end{aligned}
$$

where $t_{0}$ is defined as

$$
t_{0}=\exp \left[-\frac{\Delta\left(\Omega_{0}, \Omega\right) \tau}{k H}\right]-\exp \left[-\frac{\Delta\left(\Omega_{0}, \Omega\right) \tau_{t}}{k H}\right] .
$$

For single scattering radiances, unscattered sunlight becomes the scattering source, and the boundary conditions are determined based on the fact that no incident single scattering radiances are from above the top of atmosphere or below the bottom of the canopy. When $\tau<\tau_{a}$,

$$
\left\{\begin{array}{cl}
-\mu \frac{\partial I^{1}(\tau, \Omega)}{\partial \tau}+I^{1}(\tau, \Omega)= & \\
\frac{\omega i_{0}}{4 \pi} p\left(\Omega_{0} \rightarrow \Omega\right) \exp \left(-\frac{\tau}{\left|\mu_{0}\right|}\right) & \\
r^{1}(0, \Omega)=0 & \mu<0 \\
I^{1}\left(\tau_{a}^{b o t}, \Omega\right)=I^{1}\left(\tau_{c}^{t o p}, \Omega\right) & \mu>0
\end{array}\right.
$$

where $\tau>\tau_{a}$,

$$
\begin{cases}-\mu \frac{\partial^{-1}(\tau, \Omega)}{\partial \tau}+h(\tau, \Omega) G(\Omega) I^{1}(\tau, \Omega)= & \\ \left.\frac{i_{0}^{\prime}}{\pi}\right\urcorner\left(\Omega_{0} \rightarrow \Omega\right) \exp \left[-\left(\tau-\tau_{a}\right) \frac{G\left(\Omega_{0}\right)}{\left|\mu_{0}\right|}\right] & \\ I^{1}\left(\tau_{c}^{t}, \rho\right)=I^{1}\left(\tau_{a}^{b o t}, \Omega\right) & \mu<0 \\ I^{1}\left(\tau_{t}, \Omega\right)=0 & \mu>0\end{cases}
$$

where $i_{0}^{\prime}$ is the incident solar net flux arriving at the top of the canopy,

$$
i_{0}^{\prime}=i_{0} \exp \left(-\tau_{a} /\left|\mu_{0}\right|\right)
$$

The solutions to equations in (6) and (7) as well as boundary conditions will be separately discussed. In the downward direction $(\mu<0)$, the solution can be easily derived. When $\tau \leq \tau_{a}$

$$
\begin{aligned}
& I^{1}(\tau, \Omega)= \\
& \begin{cases}\frac{\omega F_{0} p\left(\Omega_{0} \rightarrow \Omega\right)\left|\mu_{0}\right|}{4\left(\left|\mu_{0}\right|-|\mu|\right)}\left[\exp \left(-\frac{\tau}{\left|\mu_{0}\right|}\right)-\exp \left(-\frac{\tau}{|\mu|}\right)\right] & \Omega \neq \Omega_{0} \\
\frac{\omega F_{0} \tau}{4\left|\mu_{0}\right|} P\left(\Omega_{0} \rightarrow \Omega\right) \exp \left(-\frac{\tau}{\left|\mu_{0}\right|}\right) & \Omega=\Omega_{0}{ }^{(3)}\end{cases} \\
& \text { When } \tau_{a}<\tau<\tau_{t}, \\
& I^{1}(\tau, \Omega)= \\
& \qquad \begin{array}{cc}
\frac{i_{0}^{\prime}\left|\mu_{0}\right| \Gamma\left(\Omega_{0} \rightarrow \Omega\right)}{\pi\left[G(\Omega)\left|\mu_{0}\right|-G\left(\Omega_{0}\right)|\mu|\right]} t_{1}+\Delta I^{1}(\tau, \Omega) & \Omega \neq \Omega_{0} \\
\frac{\left(\tau-\tau_{a}\right) i_{0}^{\prime} \Gamma\left(\Omega_{0} \rightarrow \Omega\right)}{\pi\left|\mu_{0}\right|} \exp \left[-G\left(\Omega_{0}\right) \frac{\tau-\tau_{a}}{\left|\mu_{0}\right|}\right] & \Omega=\Omega_{0} \\
+\Delta I^{1}(\tau, \Omega) & (9)
\end{array}
\end{aligned}
$$

where $t_{1}$ is defined as

$$
t_{1}=\exp \left[-G\left(\Omega_{0}\right) \frac{\tau-\tau_{a}}{\left|\mu_{0}\right|}\right]-\exp \left[-G(\Omega) \frac{\tau-\tau_{a}}{|\mu|}\right]
$$

and $\Delta I^{1}(\tau, \Omega)=I^{1}\left(\tau_{a}, \Omega\right) \exp \left[-G(\Omega)\left(\tau-\tau_{a}\right) /|\mu|\right]$, which is the single scattering radiance emerging from the atmosphere without further scattering in the canopy.

In the upward direction $(\mu>0)$, the solutions are a little more complicated because of the hotspot effect:

$$
\begin{aligned}
& I^{1}(\tau, \Omega)= \\
& \begin{cases}\frac{1}{\mu} \int_{\tau}^{\tau_{t}} F\left(\tau^{\prime}, \Omega\right) \\
\quad \cdot \exp \left[-\frac{1}{\mu} \int_{\tau}^{\tau^{\prime}} h(\xi, \Omega) G(\Omega) d \xi\right] d \tau^{\prime} & \tau_{a} \leq \tau \leq \tau_{t} \\
\frac{\omega F_{0} p\left(\Omega_{0} \rightarrow \Omega\right)\left|\mu_{0}\right|}{4\left(\mu+\left|\mu_{0}\right|\right)} t_{2} & \\
+I^{1}\left(\tau_{a}, \Omega\right) \exp \left(\frac{\tau-\tau_{a}}{\mu}\right) & \tau<\tau_{a}\end{cases}
\end{aligned}
$$

where $t_{2}$ is defined as

$$
t_{2}=\exp \left[-\frac{\tau}{\left|\mu_{0}\right|}\right]-\exp \left[\frac{\tau}{\mu}-\left(\frac{1}{\mu_{0}}+\frac{1}{\mu}\right) \tau_{a}\right]
$$

and the second integration at $\tau_{a} \leq \tau \leq \tau_{t}$ in the above equation can be explicitly obtained by means of (5) with an alternative integrand range, and

$$
F\left(\tau^{\prime}, \Omega\right)=\frac{i_{0}^{\prime}}{\pi} \Gamma\left(\Omega_{0} \rightarrow \Omega\right) \exp \left[-G\left(\Omega_{0}\right)\left(\tau-\tau_{a}\right) /\left|\mu_{0}\right|\right] .
$$

The radiance $I^{1}(\tau, \Omega)$ at $\tau_{a} \leq \tau \leq \tau_{t}$ needs to be numerically evaluated without further assumptions. An explicit approximation to $I^{1}(\tau, \Omega)$ has been derived and used for inversion [23]. In the following, Gauss-Legendre quadrature is employed to calculate the integration.

The source function for multiple scattering consists of an internal component which arises from the scattering of the multiple scattering component itself and two external 
components due to scattering of attenuated direct sunlight and the single scattering component. The lower boundary condition is specified based on the fact that both downward single scattering radiation and multiple scattering radiation can be reflected by the surface. The radiative transfer equations and their boundary conditions are given by

$$
\left\{\begin{array}{cl}
-\mu \frac{\partial I^{M}(\tau, \Omega)}{\partial \tau}+I^{M}(\tau, \Omega)= & \\
\quad \frac{\omega}{4 \pi} \int_{4 \pi}^{\partial \tau} p\left(\Omega^{\prime} \rightarrow \Omega\right) I^{M}\left(\tau, \Omega^{\prime}\right) d \Omega^{\prime} & \\
\quad+Q_{1}(\tau, \Omega) & \tau \leq \tau_{a} \\
-\mu \frac{\partial I^{M}(\tau, \Omega)}{\partial \tau}+G(\Omega) I^{M}(\tau, \Omega)= & \\
\frac{1}{\pi} \int_{4 \pi}^{\partial \tau} \Gamma\left(\Omega^{\prime} \rightarrow \Omega\right) I^{M}\left(\tau, \Omega^{\prime}\right) d \Omega^{\prime} & \\
+Q_{2}(\tau, \Omega) & \tau_{a}<\tau \leq \tau_{t} \\
I^{M}(0, \Omega)=0 & \mu<0 \\
I^{M}\left(\tau_{t}, \Omega\right)=\int_{2 \pi} f_{s}\left(\Omega^{\prime}, \Omega\right)\left|\mu^{\prime}\right| & \mu>0 \\
\cdot\left[I^{M}\left(\tau_{t}, \Omega^{\prime}\right)+I^{1}\left(\tau_{t}, \Omega^{\prime}\right)\right] d \Omega^{\prime} &
\end{array}\right.
$$

where

$$
\left\{\begin{array}{cc}
Q_{1}(\tau, \Omega)=\frac{\omega}{4 \pi} \int_{2 \pi_{+}} p\left(\Omega^{\prime} \rightarrow \Omega\right) & \\
\cdot I^{0}\left(\tau \cdot \Omega^{\prime}\right) d \Omega^{\prime}+\frac{\omega}{4 \pi} \int_{4 \pi} p\left(\Omega^{\prime} \rightarrow \Omega\right) & \\
\cdot I^{1}\left(\tau \cdot \Omega^{\prime}\right) d \Omega^{\prime} & \tau \leq \tau_{a} \\
Q_{2}(\tau, \Omega)=\frac{1}{\pi} \int_{2 \pi_{+}} \Gamma\left(\Omega^{\prime} \rightarrow \Omega\right) & \\
\cdot I^{0}\left(\tau \cdot \Omega^{\prime}\right) d \Omega^{\prime}+\frac{\omega}{\pi} \int_{4 \pi} \Gamma\left(\Omega^{\prime} \rightarrow \Omega\right) & \\
\cdot I^{1}\left(\tau \cdot \Omega^{\prime}\right) d \Omega^{\prime} & \tau_{a}<\tau \leq \tau_{t}
\end{array}\right.
$$

Notice that the correction function is not used in the second equation in (12), since no hotspot effect is taken into account in the multiple scattering calculation. It is obvious that no closed solution can be derived from above equations. In the following, a Gauss-Seidel iteration procedure for the coupled medium is developed to provide numerical solutions.

\section{The ImProved Gauss-Seidel Algorithm}

For simplicity of discussion, assume that we are faced with such a general equation

$$
-\mu \frac{\partial I^{M}(\tau, \Omega)}{\partial \tau}+f(\Omega) I^{M}(\tau, \Omega)=J(\tau, \Omega)
$$

where

$$
f(\Omega)= \begin{cases}1 & \tau \leq \tau_{a} \\ G(\Omega) & \tau_{a}<\tau \leq \tau_{t}\end{cases}
$$

and the source function is

$$
\begin{aligned}
& J(\tau, \Omega)= \\
& \left\{\begin{array}{cl}
\frac{w}{4 \pi} \int_{4 \pi} p\left(\Omega^{\prime} \rightarrow \Omega\right)\left[I^{M}\left(\tau, \Omega^{\prime}\right)+I^{1}\left(\tau, \Omega^{\prime}\right)\right] & \\
+\frac{\omega}{4 \pi} \int_{2 \pi_{+}} p\left(\Omega^{\prime} \rightarrow \Omega\right) I^{0}\left(\tau, \Omega^{\prime}\right) d \Omega^{\prime} & \tau \leq \tau_{a} \\
\frac{1}{\pi} \int_{4 \pi} \Gamma\left(\Omega^{\prime} \rightarrow \Omega\right)\left[I^{M}\left(\tau, \Omega^{\prime}\right)+I^{1}\left(\tau, \Omega^{\prime}\right)\right] & \\
+\frac{1}{\pi} \int_{2 \pi_{+}} \Gamma\left(\Omega^{\prime} \rightarrow \Omega\right) I^{0}\left(\tau, \Omega^{\prime}\right) d \Omega^{\prime} & \tau_{a}<\tau \leq \tau_{t} .
\end{array}\right.
\end{aligned}
$$

If the source function $J(\tau, \Omega)$ is treated independent of multiple scattering radiance $I^{M}(\tau, \Omega)$ the "formal" solution of the radiative transfer equation subject to the above boundary conditions is

$$
\left\{\begin{array}{cc}
I^{M}(\tau, \Omega)=I^{M}(0, \Omega) \exp [-f(\Omega) \tau /|\mu|] & \\
\quad+\frac{1}{|\mu|} \int_{0}^{\tau} J\left(\tau^{\prime}, \Omega\right) \exp \left[-f(\Omega)\left(\tau-\tau^{\prime}\right) /|\mu|\right] d \tau^{\prime} & \mu<0 \\
I^{M}(\tau, \Omega)=I^{M}\left(\tau_{t}, \Omega\right) \exp \left[f(\Omega)\left(\tau-\tau_{t}\right) / \mu\right] & \\
\quad+\frac{1}{\mu} \int_{\tau}^{\tau_{t}} J\left(\tau^{\prime}, \Omega\right) \exp \left[-f(\Omega)\left(\tau^{\prime}-\tau\right) / \mu\right] d \tau^{\prime} & \mu>0
\end{array}\right.
$$

The source function $J\left(\tau^{\prime}, \Omega\right)$ is related to the multiple scattering radiance $I^{M}(\tau, \Omega)$ as displayed in (16), and the coupled integral equations (17) are not the final solutions. As a result, the coupled medium is split into $N\left(N_{a}+N_{c}\right)$ contiguous layers, each of thickness $\Delta \tau_{a}$ or $\Delta \tau_{c}$ where $N_{a}$ and $N_{c}$ are the number of layers of atmosphere and canopy, respectively. From the spatial interval $\left(\tau_{i}, \tau_{i+2}\right)$, Herman and Browning [24] assume that the source function $J(\tau, \Omega)$ may be taken to be independent of $\tau^{\prime}$ and equal to its value at the midpoint of the interval, letting $J\left(\tau^{\prime}, \Omega\right)=J\left(\tau_{i+1}, \Omega\right)$ for all $\tau^{\prime}$ in this interval. But it has been proved [25], [26] that for a given choice of $\Delta \tau$, the accuracy of Herman and Browning's scheme could be improved if a linear variation of $J\left(\tau^{\prime}, \Omega\right)$ with $\tau^{\prime}$ is assumed inside the integral instead of using a value independent of $\tau^{\prime}$. For three arbitrary layers $\tau_{i} \leq \tau^{\prime} \leq \tau_{i+2}$, the linear relation can be given by:

$$
\begin{cases}J\left(\tau^{\prime}, \Omega\right)=J\left(\tau_{i}, \Omega\right) & \\ \quad+\frac{J\left(\tau_{i+1}, \Omega\right)-J\left(\tau_{i}, \Omega\right)}{\Delta \tau}\left(\tau^{\prime}-\tau_{i}\right) & \mu<0 \\ J\left(\tau^{\prime}, \Omega\right)=J\left(\tau_{i+2}, \Omega\right) & \\ \quad+\frac{J\left(\tau_{i+2}, \Omega\right)-J\left(\tau_{i+1}, \Omega\right)}{\Delta \tau}\left(\tau^{\prime}-\tau_{i+2}\right) & \mu>0\end{cases}
$$

Now we first derive the iteration formula for the downwelling direction $(\mu<0)$. From (17), it follows

$$
\begin{aligned}
I^{M}\left(\tau_{i+2}, \Omega\right)= & \exp \left[-f(\Omega) \tau_{i+2} /|\mu|\right] I^{M}(0, \Omega) \\
& +\frac{1}{|\mu|} \exp \left[-f(\Omega) \tau_{i+2} /|\mu|\right] \\
& \cdot \int_{0}^{\tau_{i+2}} J\left(\tau^{\prime}, \Omega\right) \exp \left[f(\Omega) \tau^{\prime} /|\mu|\right] d \tau^{\prime} \\
= & \exp \left[-f(\Omega) \tau_{i+2} /|\mu|\right] I^{M}(0, \Omega) \\
& +\frac{1}{|\mu|} \exp \left[-f(\Omega) \tau_{i+2} /|\mu|\right] \\
& \cdot \int_{0}^{\tau_{i}} J\left(\tau^{\prime}, \Omega\right) \exp \left[f(\Omega) \tau^{\prime} /|\mu|\right] d \tau^{\prime} \\
& +\frac{1}{|\mu|} \exp \left[-f(\Omega) \tau_{i+2} /|\mu|\right] \\
& \cdot \int_{\tau_{i}}^{\tau_{i+2}} J\left(\tau^{\prime}, \Omega\right) \exp \left[f(\Omega) \tau^{\prime} /|\mu|\right] d \tau^{\prime} \\
= & I^{M}\left(\tau_{i}, \Omega\right) \exp [-2 f(\Omega) \Delta \tau /|\mu|] \\
& +\frac{1}{|\mu|} \exp \left[-f(\Omega) \tau_{i+2} /|\mu|\right] \\
& \cdot \int_{\tau_{i}}^{\tau_{i+2}} J\left(\tau^{\prime}, \Omega\right) \exp \left[f(\Omega) \tau^{\prime} /|\mu|\right] d \tau^{\prime}(19)
\end{aligned}
$$

Replacing the source function in the above by the corresponding term in (18), we finally obtain after some algebraic 
manipulation

$$
\begin{aligned}
I^{M}\left(\tau_{i+2}, \Omega\right)== & I^{M}\left(\tau_{i}, \Omega\right) \exp \left(-2 \Delta \tau^{\prime}\right) \\
& +\frac{J\left(\tau_{i+1}, \Omega\right)}{f(\Omega)}\left[2-\frac{1-\exp \left(-2 \Delta \tau^{\prime}\right)}{\Delta \tau^{\prime}}\right] \\
& -\frac{J\left(\tau_{i}, \Omega\right)}{f(\Omega)} \\
& \cdot\left[1-1 / \Delta \tau^{\prime}+\left(1+\Delta \tau^{\prime}\right) \exp \left(-2 \Delta \tau^{\prime}\right)\right] \\
\mu & <0
\end{aligned}
$$

where $\Delta \tau^{\prime}$ is the effective optical depth of each layer in the direction $\mu$ defined by

$$
\Delta \tau^{\prime}=\frac{f(\Omega) \Delta \tau}{|\mu|}
$$

The same procedures will yield the formula for the upwelling direction

$$
\begin{aligned}
& I^{M}\left(\tau_{i}, \Omega\right)= I^{M}\left(\tau_{i+2}, \Omega\right) \exp \left(-2 \Delta \tau^{\prime}\right) \\
&+\frac{J\left(\tau_{i+1}, \Omega\right)}{f(\Omega)}\left[2-\frac{1-\exp \left(-2 \Delta \tau^{\prime}\right)}{\Delta \tau^{\prime}}\right] \\
&-\frac{J\left(\tau_{i+2}, \Omega\right)}{f(\Omega)} \\
& \cdot\left[1-\frac{1}{\Delta \tau^{\prime}}+\left(1+\Delta \tau^{\prime}\right) \exp \left(-2 \Delta \tau^{\prime}\right)\right] \\
& \mu>0
\end{aligned}
$$

For the first layer at the top and the last layer at the bottom, we have

$$
\begin{cases}I^{M}\left(\tau_{1}, \Omega\right)=J(0, \Omega)\left[1-\exp \left(-\Delta \tau^{\prime}\right)\right] & \\ \quad+I^{M I}(0, \Omega) \exp \left(-\Delta \tau^{\prime}\right) & \mu<0 \\ I^{M}\left(\tau_{N-1}, \Omega\right)=J\left(\tau_{N}, \Omega\right)\left[1-\exp \left(-\Delta \tau^{\prime}\right)\right] & \\ \quad+I^{M I}\left(\tau_{N}, \Omega\right) \exp \left(-\Delta \tau^{\prime}\right) & \mu>0 .\end{cases}
$$

According to Herman and Browning's assumption about the source function within the medium, the iteration formula corresponding to (20) and (22) can be written as

$$
\left\{\begin{array}{cc}
I^{M}\left(\tau_{i+2}, \Omega\right)=I^{M}\left(\tau_{i}, \Omega\right) \exp \left(-2 \Delta \tau^{\prime}\right) & \\
\quad+J_{\left(\tau_{i+1}, \Omega\right)}\left[1-\exp \left(-2 \Delta \tau^{\prime}\right)\right] & \mu<0 \\
f(\Omega) & \\
I^{M}\left(\tau_{i}, \Omega\right)=I^{M}\left(\tau_{i+2}, \Omega\right) \exp \left(-2 \Delta \tau^{\prime}\right) & \\
\quad+\frac{\left.J_{i+1}, \Omega\right)}{f(\Omega)}\left[1-\exp \left(-2 \Delta \tau^{\prime}\right)\right] & \mu>0 .
\end{array}\right.
$$

The angular variables can be discretized by choosing a finite set of $M$ directions using Gauss-Legendre quadrature in the unit sphere, and applying the Gauss-Seidel iteration technique as follows. Start at the top of the atmosphere and work downwards at successive interfaces using (20) or (24) until the lower boundary (soil) is reached. Reflection of the background given at the bottom (12) provides the upwelling radiance, and so work upwards at successive interfaces using (22) or (24) until the upper boundary is reached. At this state, update the source function based on (16). This downwards and upwards cycling is continued until convergence is obtained.

One comment on this algorithm is about its parallel property. From (20) and (22) or (24), it can be shown that for every discrete direction $\left(\Omega_{i j}\right)$ the iteration formulas are identical and independent of each other. If multiple processors are available, such as a Connection Machine or other parallel supercomputer, each processor can manipulate the iteration for a specific angle $\Omega_{i j}$, and the calculation time will be dramatically reduced. This is highly desired because solving such a radiative transfer equation is very time consuming.

Further, after calculating the downward radiance of the bottom layer, the upward radiances are calculated based on the bottom reflectance function, which can be of any form. Thus, a non-Lambertian boundary condition can be used without extra computational expense. However, if we use the discreteordinate approach, the non-Lambertian reflectance function usually needs to be expanded in Legendre polynomials [27]. If the number of terms of the Legendre polynomials, which is equal to the number of streams of the discrete-ordinate method, is not large enough, the constructed directional function wotld not represent the actual reflectance well.

\section{NUMERICAL IMPLEMENTATION AND MODEL VALIDATION}

The Gauss-Seidel algorithm, like most other numerical techniques, restricts photon travel to discrete directions. The lack of rotational invariance precludes the use of Legendre polynomial expansions and the addition theorem for the spherical harmonics expansion of the argument of the Legendre polynomials [6]. Thus, the exact kernel method [6], [17], [28] is applied in this study, and the scattering function is evaluated directly for transfer between all possible directions $\left(\Omega_{m n} \rightarrow \Omega_{i j}\right)$. The major drawback of this method is that much higher storage and computation are required if high angular resolution is desired. Some symmetry relations [6], [8] are quite useful in overcoming this problem:

$$
\left\{\begin{array}{l}
\Gamma\left(\Omega^{\prime} \rightarrow \Omega\right)=\Gamma\left(\Omega \rightarrow \Omega^{\prime}\right) \\
\Gamma\left(\Omega^{\prime} \rightarrow \Omega\right)=\Gamma\left(-\Omega^{\prime} \rightarrow \Omega\right) \\
\Gamma\left(\Omega^{\prime} \rightarrow \Omega\right)=\Gamma\left(\mu, \mu^{\prime}, \phi-\phi^{\prime}\right) \\
\Gamma\left(\mu, \mu^{\prime}, x\right)=\Gamma\left(\mu, \mu^{\prime},-x\right)=\Gamma\left(\mu, \mu^{\prime}, x+2 \pi\right) .
\end{array}\right.
$$

All integrations in the formulae shown in the previous section are numerically calculated by using Gauss-Legendre quadratures. If we are interested in radiances in specific directions instead of double-Gauss discrete directions, (17) can be applied to obtain radiance at arbitrary angles by integrating the source function. This procedure is sometirnes referred to as the iteration of the source-function technique, but essentially amounts to an interpolation [27]. Another way to calculate the radiance at arbitrary directions is to apply (19) and (20) after the source function has been calculated based on Gauss-Legendre quadratures.

In our computations, a pointwise convergence criteria [16] of 0.002 is used, and the coupled medium is divided so that the optical depth of each layer is about $0.08-0.20$. Experiments show that the iteration could be unstable if the optical depth of each layer is greater than 0.2 . 
TABLE II

Validation of Gauss-Seidel Algorithm Using Discrete-Ordinate Algorithm

\begin{tabular}{|c|c|c|c|c|c|c|}
\hline \multirow{3}{*}{$\begin{array}{l}\text { viewing angles } \\
\qquad \mu=\cos \theta\end{array}$} & \multicolumn{6}{|c|}{ upwelling radiance } \\
\hline & \multicolumn{2}{|c|}{$\tau=0.1$} & \multicolumn{2}{|c|}{$\tau=1.0$} & \multicolumn{2}{|c|}{$\tau=3.0$} \\
\hline & Gauss -Seidel & $\begin{array}{l}\text { Discrete- } \\
\text { Ordinate }\end{array}$ & Gauss - Seidel & Discrete-Ordinate & Gauss - Seidel & Discrete-Ordinate \\
\hline 0.067 & 0.43419 & 0.43408 & 0.59566 & 0.59400 & 0.62154 & 0.62237 \\
\hline 0.283 & 0.25950 & 0.25955 & 0.47818 & 0.47954 & 0.52799 & 0.53298 \\
\hline 0.574 & 0.22143 & 0.22146 & 0.32526 & 0.32689 & 0.39136 & 0.39783 \\
\hline 0.840 & 0.21233 & 0.21234 & 0.24526 & 0.24652 & 0.29265 & 0.29904 \\
\hline 0.987 & 0.20962 & 0.20963 & 0.21330 & 0.21441 & 0.24804 & 0.24803 \\
\hline
\end{tabular}

The program has been tested using many datasets, two of which will be illustrated here. One compares with the upwelling radiances of a aerosol atmosphere calculated by DISORT based on a discrete ordinate algorithm [27]. The results using 20 by 20 Gauss-Legendre directions in the Gauss-Seidel code and 32 streams in discrete directions in DISORT code are compared in Table II, where $F_{0}=1.0$, $G=0.65, W=0.96, \theta_{0}=45.3^{\circ}, \phi_{0}=0^{\circ}, R_{s}=0.3$. From the table, we can see that the relative error is about $0.1 \%-0.5 \%$. The improved algorithm is not sensitive to the optical depth of each layer, but quite sensitive to the number of discrete directions. The larger the number of the discrete directions, the smaller the relative error.

Another validation was carried to test the formulation of the canopy radiative transfer model. Marshak [8] compared this model with a Monte Carlo simulation [29] in the visible region using a discrete ordinate numerical method. The same formulation and parameters are used but with different numerical algorithms. The reflectance is the upwelling radiance normalized by cosine of the solar zenith angle:

$$
R\left(\Omega_{0}, \Omega\right)=\frac{\pi I(0, \Omega)}{\left|\mu_{0}\right|}
$$

where the incident flux has been assumed unity. Fig. 2 shows that our results are basically equivalent to Marshak's for the specific set of parameters. In this case, the multiple scattering radiance is small, but the main purpose of this calculation is to validate the formulation and the single scattering calculation, especially for the hotspot effect.

\section{NUMERICAL SOlUTION AND DATA ANALYSIS}

For this analysis, two wavelengths were selected: $0.65 \mu \mathrm{m}$ standing for the red band and $0.90 \mu \mathrm{m}$ standing for the nearIR band. The Rayleigh optical depth is calculated based on the formula

$$
\tau_{r}=0.0088 \lambda^{0.2 \lambda-4.15}(\lambda \text { in } \mu \mathrm{m})
$$

and aerosol optical depths are taken based on different atmosphere visibilities [30]. The canopy parameters are taken from the literature [8], [31], and are discussed further in the following paragraphs. The extraterrestrial solar incident net flux at the top of atmosphere is always assumed to be $\pi$ (i.e., $F_{0}=1.0$ ).

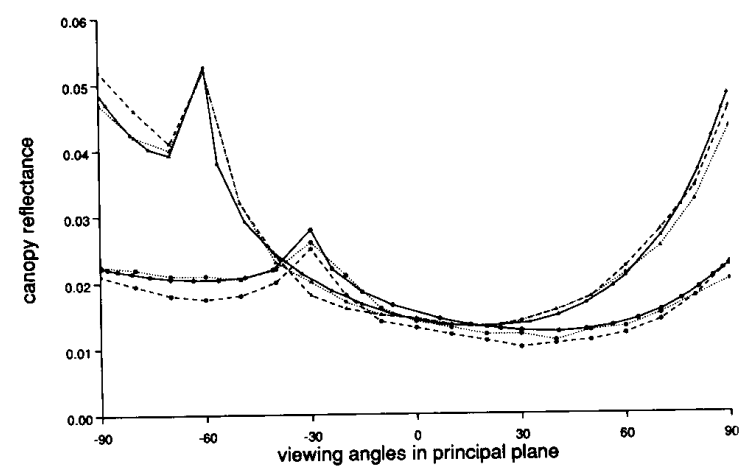

Fig. 2. Comparison of the canopy reflectances calculated by the GaussSeidel algorithm with the discrete ordinate algorithm and the Monte Carlo simulation in the visible band. Solar zenith angles are $\theta_{0}=30^{\circ}$ and $\theta_{0}=60^{\circ}, r_{l}=t_{l}=0.04, R_{s}=0.0, n=1.0, k=0.08, L A I=4.0$. The leaf orientation is uniform (spherical). Solid lines stand for our results, dashed lines for the Monte Carlo method, and dotted lines for the discrete ordinate method.

\section{A. Atmosphere Perturbation}

The upwelling radiances with different aerosol optical depths above both atmosphere and canopy in the visible and nearIR regions are illustrated in Fig. 3. The optical parameters of canopy leaves are taken from data measured by Ranson et al. [32]. In the visible region, the atmosphere largely masks the hotspot, and the angular distribution of radiance above the atmosphere mainly reflects the atmospheric path radiance characteristics. The forward scattering peaks are also detectable. In the near-IR region, however, the canopy has a higher reflectivity and aerosol optical depth becomes much smaller. Thus the radiances received by sensors above the atmosphere basically depend on the canopy radiation field. Large attenuation occurs in the large viewing angles in the backscattering direction.

\section{B. Effects of Canopy Roughness Coefficient $k$}

Fig. 4 illustrates the influence of the canopy roughness coefficient $k$, which characterizes the leaf dimensions in the radiation field of the coupled medium. The parameters are shown in the figure caption. As stated previously, the parameter $k$ is directly proportional to the ratio of leaf diameter to canopy height. When the leaf is infinitesimal $(k=0)$, no shadows occur and no hotspot peak is observed. As $k$ becomes larger, 
red

over atmosphere
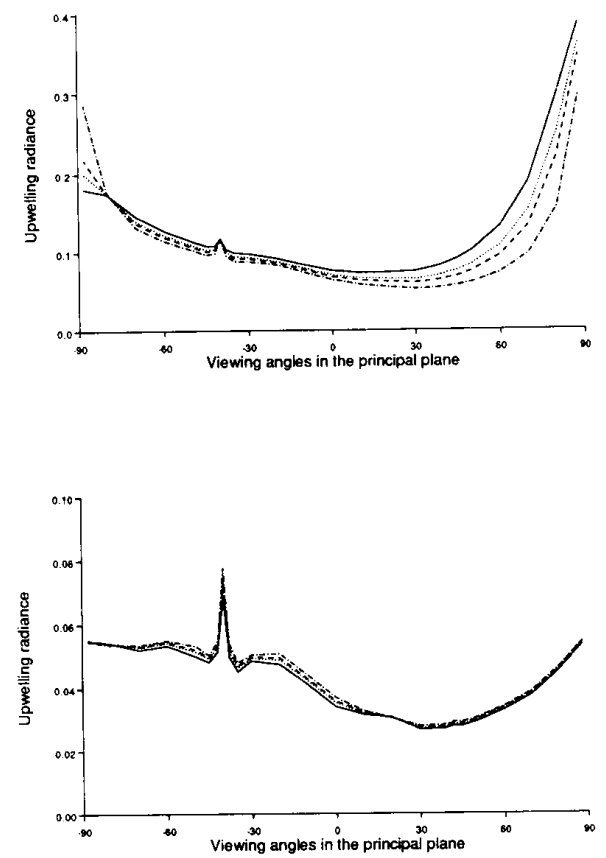

near-IR
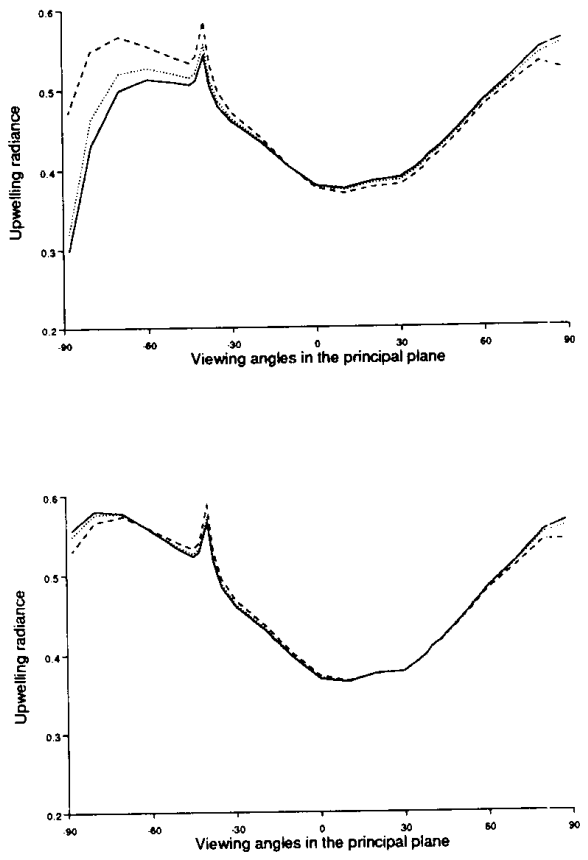

Fig. 3. Upwelling radiances above both canopy and atmosphere at different aerosol optical depths. $\theta_{0}=40^{\circ}, k=0.08, n=1.2, L A I=3.0$, ind the leaf orientation is erectophile. In the near-IR region, $r_{l}=0.454, t_{l}=0.518, R_{s}=0.23$, three curves stand for three aerosol optical thicknesses: 0.01 (dashed line), 0.1 (dotted line), and 0.15 (solid line). For the visible region, $r_{l}=0.0878, t_{l}=0.932, R_{s}=0.13$, four curves stand for four aerosol optical thicknesses: 0.01 (dot-dashed line), 0.1(dashed line), 0.15 (dotted line), and 0.25 (solid line).

upwelling radiance increases, mainly in near-hotspot region and the width of the hotspot peak goes up as well. This implies that other viewing directions are not sensitive to the variation of leaf sizes. Notice that the heights of the hotspot peak remain constant, since soil reflectance and other optical parameters do not vary. The dependence of reflectance of the canopy on $k$ at visible wavelength is evaluated by Marshak [8].

\section{Effect of Leaf Area Index (LAI)}

LAI is of great interest for many applications of remote sensing. Fig. 5 is an illustration of the upwelling radiance of canopy at different LAI levels. In the near-IR region, when LAI increases, the upwelling radiances increase correspondingly, but the angular dependence remains about the same. This observa:ion implies that LAI retrieval is not enhanced by fine resolution in view angles. This fact also shows that the LAI variation may cause large errors if the canopy is treated as a semi-infinite medium [2] [33]. Note that the upwelling radiance in the visible region above the atmosphere does not vary significantly with increasing LAI if LAI is greater than 3.0. Thus it will be quite difficult to retrieve large LAI values using visible bands.

\section{Effect of l eaf Angle Distribution (LAD)}

The effect of leaf orientation on the canopy radiation field has be in investigated for many years. In the earliest approaches, such as the Suits model [34], only projected horizontal and vertical components of leaf orientation were considered. Further efforts explicitly incorporated the leaf orientation angle in models [35]-[37]. LAD is parameterized by the leaf inclination function $g_{l}\left(\mu_{l}\right)$. Different theoretical and experimental models for this function have been published, such as elliptical [38] or beta distributions [39]. Here the triangular formulae of Bunnik [40], based on the data of de Wit [41], are used: $g_{l}\left(\theta_{l}\right)=\frac{2}{\pi}+b \cos 2 \theta_{l}+c \cos 4 \theta_{t}$, where parameters $b$ and $c$ characterize different canopies. For example, for a planophile canopy (mainly horizontal leaves), $b=\frac{2}{\pi}$ and $c=0$; for an erectophile canopy (main vertical leaves), $b=-\frac{2}{\pi}$ and $c=0$; for a plagiophile canopy (mainly leaves with $45^{\circ}$ inclination angles), $b=0$ and $c=-\frac{2}{\pi}$; and for a uniform (spherical) canopy (random distribution), $b==0$ and $c=0$.

The results for these four LADs in the near-IR region are illustrated in Fig. 6. From the figure, it can be observed that not all of viewing angles can distinguish different LADs. Therefore, the selection of the optimum viewing angles becomes important. For these examples, a comparison of nadir response with the response at $+75^{\circ}$ (forward scattering) would be most effective.

\section{E. Multiple and Single Scattering}

The significance of the multiple scattering component as 

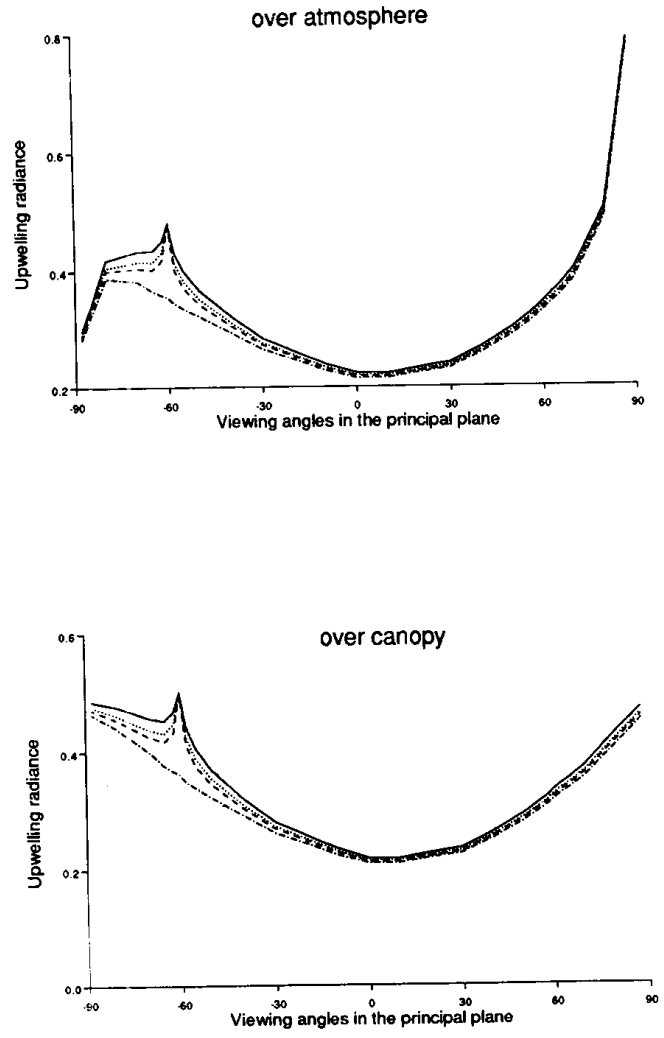

Fig. 4. Evaluation of the effect of the canopy roughness coefficient $k$ on upwelling radiance above both canopy and atmosphere in the near-IR region: $\tau_{a e}=0.05, \omega=0.92, r_{l}=0.46, t_{l}=0.46, R_{s}=0.3 . \theta_{0}=60^{\circ}$ $n=1.2, L A I=3.0$, and the leaf orientation is erectophile. The four curves stand for four $k$ values: 0.0 (dot-dashed line), 0.05 (dashed line), 0.08 (dotted line), and 0.15 (solid line)

compared to single scattering has been analyzed by Bohren for the atmosphere [42]. Otterman and Brakke examined the canopy case, but under somewhat restricted conditions [43]. Our formulation for solving the radiative transfer equations allows straightforward analysis of the relative magnitudes of single scattering and multiple scattering components. Fig. 7 illustrates the ratio of the upwelling multiple scattering radiance to the total upwelling radiance in the red and near-IR regions. The mean value of the ratio of multiple scattering to total scattering radiance in the principal plane is $19.6 \%$ and $29.2 \%$ above the canopy and atmosphere in the visible region, and $52.0 \%$ and $53.8 \%$ in the near-IR region, respectively.

\section{DISCUSSION AND CONCLUSION}

The radiative transfer equations for a coupled atmospherecanopy system and its boundary conditions are presented in this paper. The radiation field of the coupled medium is analyzed in the context of three components: unscattered sunlight, single scattering and multiple scattering radiance. The first two components have analytical solutions, but the multiple scattering radiance has no closed-form solution, and an improved Gauss-Seidel algorithm is used to derive the iteration formula.
This study shows that the angular characteristics of upwelling canopy radiance in the visible region are heavily masked by the atmosphere. However, in the near-IR region, the radiance above the atmosphere mainly depends on canopy biophysical parameters and the configuration of the canopy. Therefore, the near-IR bands are of great significance for potential inversion of radiance obtained from the multiangle satellite imagery. The upwelling radiance is quite sensitive to the leaf size and leaf angle distribution in some viewing regions. Thus, selection of optimum viewing angles is important if inference is desired. Variation in leaf area index mainly affects the absolute magnitude of the upwelling radiance, and the angular characteristics do not significantly vary. In the visible region, the upwelling radiance does not significantly vary for different LAI's if LAI is larger than 3.0. The numerical comparisons of single scattering and multiple scattering components indicate that an oversimplified calculation of the multiple scattering component will result in a large error in the prediction of directional reflectance of the atmosphere and/or canopy system. Finally, we have already noted that the Gauss-Seidel algorithm lends itself quite easily to computation on a Connection Machine or other parallel supercomputer.

\section{APPENDIX I \\ ATMOSPHERE AND CANOPY RADIATIVE TRANSFER EQUATIONS}

\section{A. Atmospheric Radiative Transfer Model}

For a plane-parallel homogeneous atmosphere in the absence of polarization, the radiative transfer equation can be written as [44]

$$
\mu \frac{\partial I(\tau, \Omega)}{\partial \tau}=I(\tau, \Omega)-\frac{\omega}{4 \pi} \int_{4 \pi} p\left(\Omega^{\prime} \rightarrow \Omega\right) I\left(\tau, \Omega^{\prime}\right) d \Omega^{\prime}
$$

where $\omega$ is the single scattering albedo, and $\tau$ is the optical depth varying from zero at the top of the atmosphere to $\tau_{a}$ above canopy. The solid angle $\Omega(\mu, \phi)$ has the azimuthal angle $\phi$ and a zenith angle $\theta=\cos ^{-1}(\mu)$.

The scattering properties of the atmosphere depend on Rayleigh and aerosol particles. Thus, the scattering phase function can be defined as a weighted average of individual scattering phase functions at specific scattering angles:

$$
p(\Psi)=\frac{p_{r}(\Psi) \tau_{r}+p_{a}(\Psi) \tau_{a e}}{\tau_{r}+\tau_{a e}}
$$

with the constraint $\frac{1}{2} \int_{0}^{\pi} p(\Psi) \sin \Psi d \Psi=1$. Here $\Psi$ is the scattering angle dependent on solar zenith angle $\theta_{0}=$ $\cos ^{-1} \mu_{0}$, viewing angle $\theta$, and angle difference between solar azimuth and viewing azimuth $\phi-\phi_{0} . \tau_{r}$ and $\tau_{a e}$ are the molecular optical depth and aerosol optical depth, respectively. The One-Term Henyey-Greenstein (OTHG) function is used as the aerosol phase function. It does underestimate the forward scattering so that the reflected radiance will be underestimated a little bit, but our primary calculations using both Mie phase function and Henyey-Greenstein function show that the approximation does not obviously affect the angular patterns of the radiance distribution. The asymmetry parameter 0.65 has been assumed in all our calculations. For simplicity, 
Red

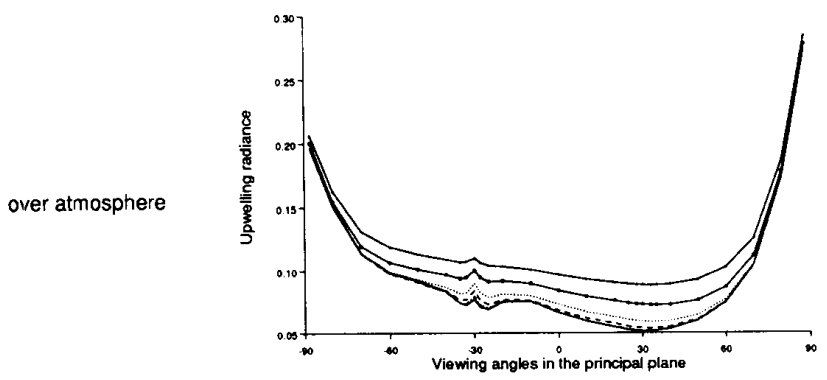

over canopy
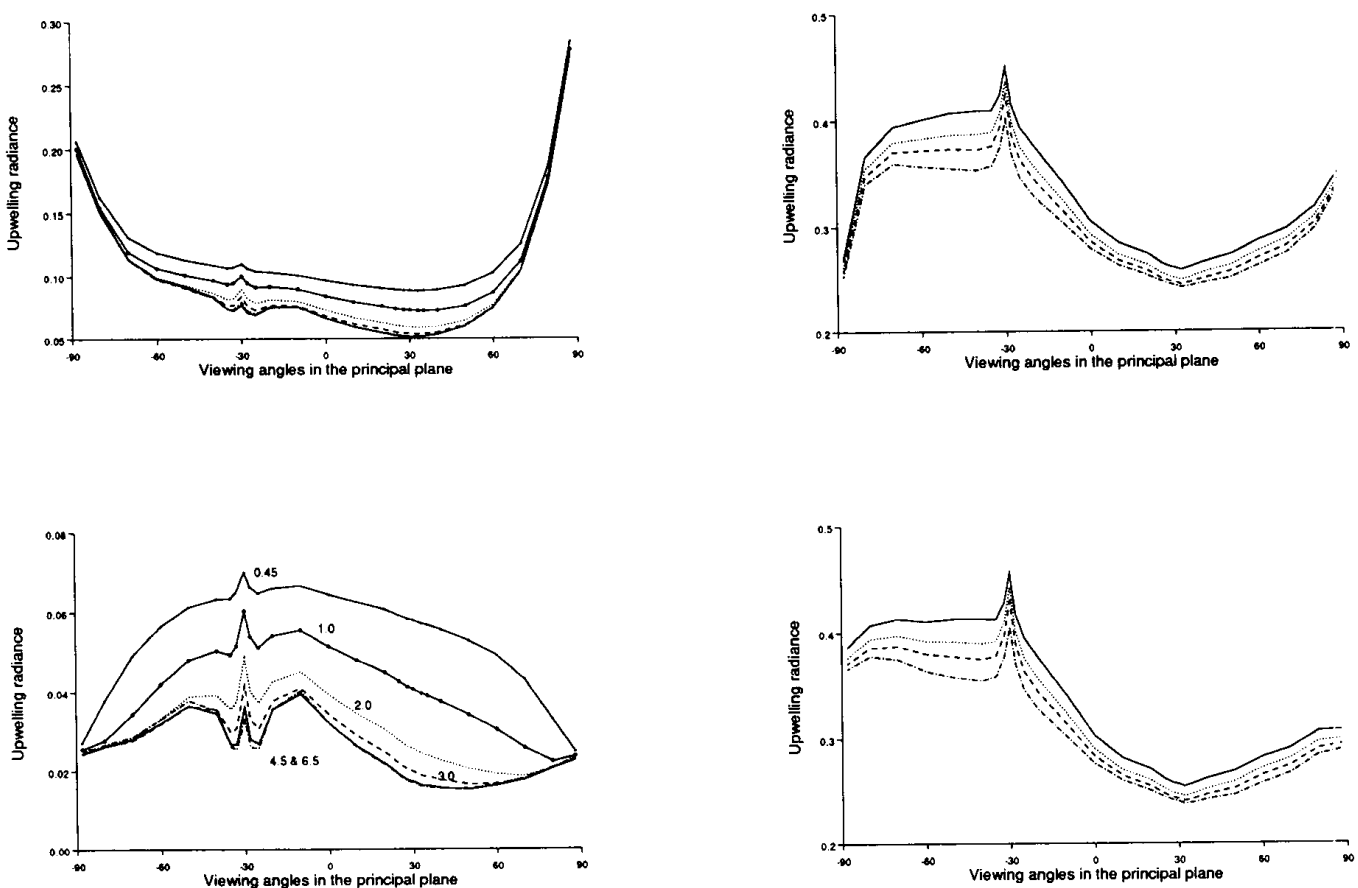

Fig. 5. Evaluation of the effect of canopy LAI on upwelling radiance above both canopy and atmosphere. $\theta_{0}=30^{\circ}, k=0.08, n=1.2$, and the leaf orientation is erectophile. In the near-IR region, $\tau_{a e}=0.05, \omega=0.92, r_{l}=0.5, t_{l}=0.3, R_{s}=0.3$. Four curves stand for four LAI values: 2.0(dot-dashed line), 3.0(dashed line), 4.0(dotted line), and 6.5(solid line). In the visible region, $\tau_{a e}=0.1, \omega=0.96, r_{l}=0.04, t_{l}=0.04, R_{s}=0.1$. Five curves stand for five LAI values: $0.45,1.0,2.0,3.0,4.5$, and 6.5 .

it is assumed that only molecules and aerosols are included in the atmosphere. Aerosols are treated as absorbing as well as scattering particles; all the cases with single scattering albedo $\omega=0.96$ for visible region and $\omega=0.92$ for near-IR region are examined.

To obtain is solution of (A1), appropriate boundary condilions must be specified. On the upper boundary, the atmosphere is illuminated by a parallel beam in the direction $\left(\Omega_{0}\right)$ with net flux $i_{0}=\pi F_{0}$, i.e.,

$$
I(0, \Omega)=\delta\left(\Omega-\Omega_{0}\right) i_{0}
$$

where $\mu<0$. For the coupled medium, the lower boundary condition at the bottom of the canopy will be discussed in the following section.

\section{B. Canopy Radiative Transfer Model}

The one-d mensional radiative transfer equation of a flat homogeneous canopy is given by [6]:

$$
\begin{aligned}
&-\mu \frac{\partial I(\tau, \Omega)}{\partial \tau}+h(\tau, \Omega) G(\Omega) I(\tau, \Omega)= \\
& \frac{1}{\pi} \int_{4 \pi} \Gamma\left(\Omega^{\prime} \rightarrow \Omega\right) I\left(\tau, \Omega^{\prime}\right) d \Omega^{\prime}
\end{aligned}
$$

with the boundary condition

$$
I(\tau, \Omega)=\int_{2 \pi_{-}} f_{s}\left(\Omega^{\prime}, \Omega\right)\left|\mu^{\prime}\right| I\left(\tau_{t}, \Omega^{\prime}\right) d \Omega^{\prime}
$$

for $\mu>0$, where $f_{s}\left(\Omega^{\prime}, \Omega\right)$ is the directional reflectance distribution function (BRDF) of background (e.g., soil) under the canopy, and $2 \pi_{-}$stands for the lower hemisphere. For an almost Lambertian surface with reflectance $R_{s}$, the above boundary condition can be written as

$$
I\left(\tau_{t}, \Omega\right)=\frac{R_{s}}{\pi} \int_{2 \pi_{-}} I\left(\tau_{t}, \Omega^{\prime}\right)\left|\mu^{\prime}\right| d \Omega^{\prime}
$$

$G(\Omega)$ is a geometry factor defined later, $h(\tau, \Omega)$ is an empirical correction function for accounting for the variation of extinction coefficient [8], [45], which will be discussed in more detail later.

The function $G(\Omega)$ is the mean projection of a unit foliage area in the direction $\Omega$, i.e.

$$
G(\Omega)=\frac{1}{2 \pi} \int_{2 \pi_{+}} g_{l}\left(\Omega_{l}\right)\left|\Omega_{l} \cdot \Omega\right| d \Omega_{l}
$$

where $2 \pi_{+}$stands for the upper hemisphere. $g_{l}\left(\Omega_{l}\right)$ is the probability density of the distribution of the leaf normals with respect to the upper hemisphere. It is assumed that the zenith and azimuthal angles of the distribution of the leaf normals are independent and the distribution in the azimuth is uniform, that is $g_{l}\left(\Omega_{l}\right) \equiv g_{l}\left(\mu_{l}\right)$. More explicit formulas have been evaluated by Shultis and Myneni [6].

In (A2), the area scattering phase function $\Gamma\left(\Omega^{\prime} \rightarrow \Omega\right)$, consisting of both diffuse and specular components, is defined 

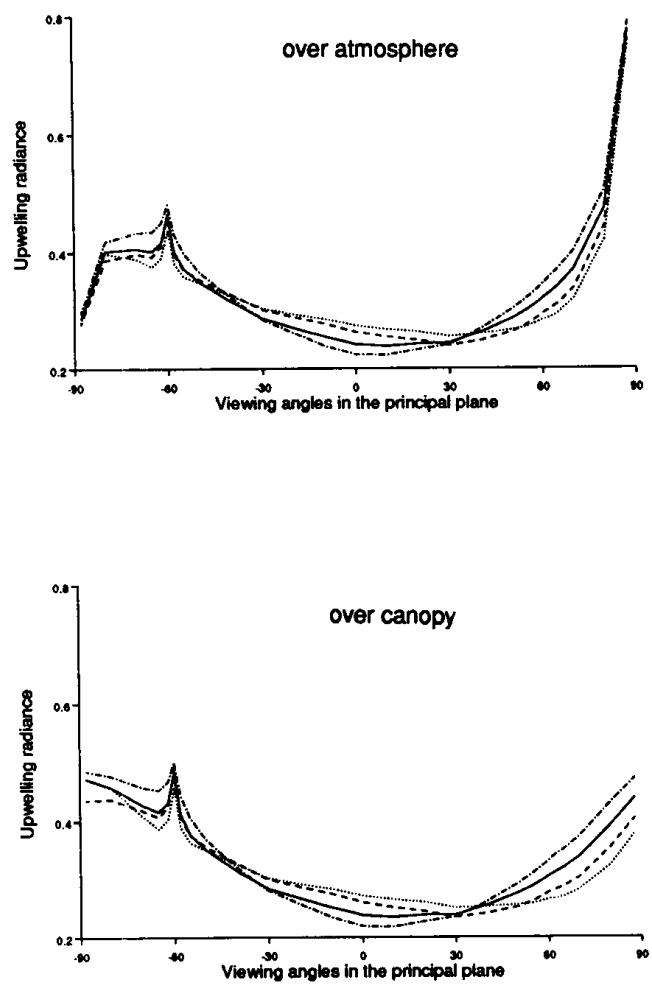

Fig. 6. Evaluation of the effect of canopy $L A D$ on upwelling radiance in the near-IR region. $\theta_{0}=60^{\circ}, k=0.08, n=1.2, L A I=3, \tau_{a e}=0.05$ $\omega=0.92, r_{l}=0.46, t_{l}=0.46, R_{s}=0.3$. Solid lines stand for uniform canopy, dotted lines for planophile, dashed lines for plagophile, and dot-dashed lines for erectophile.

as:

$$
\Gamma\left(\Omega^{\prime} \rightarrow \Omega\right)=\Gamma_{D}\left(\Omega^{\prime} \rightarrow \Omega\right)+\Gamma_{s p}\left(\Omega^{\prime} \rightarrow \Omega\right) .
$$

We will find that $\Gamma\left(\Omega^{\prime} \rightarrow \Omega\right)$ depends on not only the scattering angle between $\Omega^{\prime}$ and $\Omega$, but also on the absolute value of $\Omega^{\prime}$ and $\Omega$. It is assumed that the diffuse scattering for the leaves follows the bi-Lambertian scattering model, giving

$$
\begin{aligned}
\Gamma_{D}\left(\Omega^{\prime} \rightarrow \Omega\right)= & \frac{1}{2 \pi} \int_{\Omega^{+}} g_{l}\left(\Omega_{l}\right) t_{l} \alpha^{\prime} \alpha d \Omega_{l} \\
& -\frac{1}{2 \pi} \int_{\Omega^{-}} g_{l}\left(\Omega_{l}\right) r_{l} \alpha^{\prime} \alpha d \Omega_{l} .
\end{aligned}
$$

Here $\Omega^{+}, \Omega^{-}$indicate that the $\Omega_{l}$ integration is over that portion of the $0-2 \pi$ range for which the integrand is either positive $(+)$ or negative $(-)$. In this model, a fraction $r_{l}$ of the intercepted energy is radiated in a cosine distribution about the leaf normal (i.e., Lambertian reflectance). Similarly, a fraction $t_{l}$ is transmitted in a Lambertian distribution on the opposite side of the leaf. It is obvious that $\Omega^{+}, \Omega^{-}$is a part of the hemisphere for which $\pm \alpha^{\prime} \alpha>0, \alpha^{\prime}=\Omega^{\prime} \cdot \Omega_{l}, \alpha=\Omega \cdot \Omega_{l}$.

The area phase function of specular component $\Gamma_{s p}\left(\Omega^{\prime} \rightarrow\right.$ $\Omega$ ) can be evaluated as [8]

$$
\Gamma_{s p}\left(\Omega^{\prime} \rightarrow \Omega\right)=\frac{1}{8} g_{l}\left(\Omega_{l}^{*}\right) K\left(\kappa, \Omega^{\prime} \times \Omega_{l}^{*}\right) F\left(n, \Omega^{\prime} \cdot \Omega_{l}^{*}\right)
$$
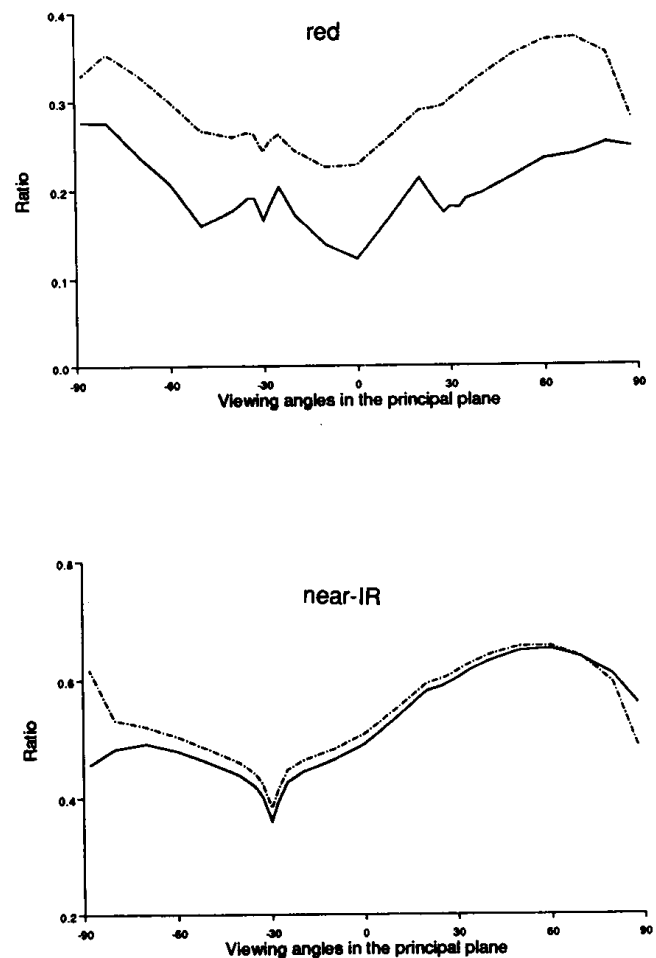

Fig. 7. Ratios of multiple-scattering to single-scattering radiance in the upwelling direction above both canopy (solid line) and atmosphere (dot-dashed line) in both visible and near-IR regions. The parameters are the same as Fig. 5.

where $\Omega_{l}^{*}=\Omega_{l}^{*}\left(\Omega^{\prime} \rightarrow \Omega\right)$ defines the direction of the appropriate leaf normal for specular scattering between the incident and the reflected rays. It has been shown that [46]

$$
\left\{\begin{array}{l}
\mu_{l}^{*}=\frac{\mu_{i}+\mu}{2 \mu_{\gamma}} \\
\cos \phi_{l}^{*}=\frac{\sqrt{1-\mu_{l}^{2}}+\sqrt{1-\mu^{2}} \cos \left(\phi-\phi_{i}\right)}{2 \mu_{\gamma} \sqrt{1-\mu_{l}^{* 2}}}
\end{array}\right.
$$

where

$$
\mu_{\gamma}=\sqrt{\frac{\mu_{i} \mu+\sqrt{1-\mu_{i}^{2}} \sqrt{1-\mu^{2}} \cos \left(\phi-\phi_{i}\right)+1}{2}} .
$$

The term $f\left(n, \alpha^{\prime}\right)$ is the Fresnel reflectance, indicating the amount of specularly reflected energy for incident unpolarized radiance:

$$
F\left(n, \alpha^{\prime}\right)=\frac{1}{2}\left[\frac{\sin ^{2}(j-i)}{\sin ^{2}(j+i)}+\frac{\tan ^{2}(j-i)}{\tan ^{2}(j+i)}\right]
$$

where $j=\cos ^{-1}\left(\left|\alpha^{\prime}\right|\right), i=\sin ^{-1}\left(\sqrt{1-\alpha^{\prime 2}} / n\right), n$ is the wax refractive index of canopy leaves. If the leaf is optically smooth and flat on a microscale, the Fresnel reflectance law is enough to compute the amounts of specularly reflected radiance, besides considering the number of leaves contributing to specular reflectance $g_{l}\left(\Omega_{l}^{*}\right)$. However, a leaf wax surface is rarely optically smooth, and the hair structure on the leaf surface reflects light diffusely, producing incompletely specular reflectance. Therefore, a smoothing factor $K$ is defined to 
account for this reduction in the amount of specularly reflected light [47]. Nilson and Kuusk [45] give a form of this factor:

$$
K\left(\kappa, \alpha^{\prime}\right)=\exp \left[-2 \kappa \tan \left(\alpha^{\prime}\right) / \pi\right]
$$

where $\alpha^{\prime}=\cos ^{-1}\left(\Omega^{\prime} \cdot \Omega_{l}\right)$. The argument $\kappa \geq 0$ characterizes the dimension of the hair on the leaf surface. $\kappa=0.3$ has been used in this paper.

The area scittering phase function defined above is required to meet the normalization condition [8]

$$
\frac{1}{\pi} \int_{4 \pi} \frac{\Gamma\left(\Omega^{\prime} \rightarrow \Omega\right)}{\tilde{G}\left(\Omega^{\prime}\right)} d \Omega=1
$$

where

$$
\begin{aligned}
\tilde{G}\left(\Omega^{\prime}\right)= & \frac{1}{2 \pi} \int_{2 \pi+} g_{l}\left(\Omega_{l}\right)\left|\alpha^{\prime}\right| \\
& \cdot\left[r_{l}+t_{l}+K\left(\kappa, \alpha^{\prime}\right) F\left(n, \alpha^{\prime}\right)\right] d \Omega_{l} .
\end{aligned}
$$

The correction function $h(\tau, \Omega)$ in (A2) is used to account for hotspol phenomena. Such functions have been discussed in detail by J גpp and Strahler [48]. Here we use Nilson and Kuusk's formulation [45], for unscattered solar radiation and single scattering radiation in the upwelling directions $(\mu>0)$. It is given by

$$
h(\tau, \Omega)=1-\sqrt{\frac{G\left(\Omega_{0}\right)}{G(\Omega)} \frac{\mu}{\left|\mu_{0}\right|}} \exp \left[-\frac{\Delta\left(\Omega_{0}, \Omega\right) \tau}{k H}\right]
$$

otherwise $h,(\tau, \Omega)=1$, where $\Delta\left(\Omega_{0}, \Omega\right)=$ $\sqrt{\mu_{0}^{-2}+\mu^{-2}+2\left(\Omega_{0} \cdot \Omega\right) /\left|\mu_{0} \mu\right|}$. The parameter $k$ characterizes the dimension of the leaf and is proportional to the ratio of the averace diameter of round leaf to the canopy height $H$. Notice thit in the case of backscattering (i.e., $\Omega=-\Omega_{0}$ ), $\Delta\left(\Omega_{0}, \Omega\right)=0$ and $h(\tau, \Omega)=0$. The absence of extinction results in the local maximum of reflectance which is widely termed the hotspot peak.

Notice that the correction function $h(\tau, \Omega)$ becomes smaller than unity or ly for unscattered sunlight and single scattering components : $n$ the upwelling direction. Esposito's numerical calculation [49] shows that including a hotspot effect in higher order scattering than single scattering has a negligible effect.

\section{ACKNOWLEDGMENT}

The autho's are indebted to Dr. Yoram Kaufman for his thoughtful ccimments on the manuscript, and to Dr. Xiaowen Li for his careful review of an early draft of this paper. Dr. W. J. Wiscombe kindly provided the radiative transfer code DISORT.

\section{REFERENCES}

[1] J. R. Irons, K. J. Ranson, D. L. Williams, R. R. Irish and F. G. Huegel, "An off-nadir pointing imaging spectroradiometer for terrestrial ecosystem studies," IEEE Trans. Geosci. Remote Sensing, vol. 29, pp. 66-74, 1991.

[2] J. Ross, The Radiation Regime and Architecture of Plant Stands. The Hague: D: W. Junk, 1981.

[3] R. B. Myreni, A. L. Marshak, and Y. V. Knyazikhin, "Transport theory for a leaf canopy of finite-dimensional scattering centers," J. Quant. Spectrosc. Radiat. Transfer, vol. 46, pp. 259-280, 1991.
[4] Y. V. Knyazikhin, A. L. Marshak, and R. B. Myneni, "Interaction of photons in a canopy of finite-dimensional leaves," Remote Sens. Enviorn., vol. 39, pp. $61-74,1992$.

[5] J. Ross and T. Nilson, "A mathematical model of radiation regime of plant cover," in Actinometry and Atmospheric Optics, Valgus publ. Tallinn, Estonia, pp. 263-281, 1968

[6] J. K. Shultis and R. B. Myneni, "Radiative transfer in vegetation canopies with an isotropic scattering," J. Quant. Spectrosc. Radiat. Transfer, vol. 39 , pp. $115-129,1988$.

[7] V.P. Gutschick and F.W. Weigel, "Radiative transfer in vegetative canopies and other layered media: Rapidly solvable exact integral equation not requiring Fourier resolution," J. Quant. Spectrosc. Radiat. Transfer, vol, 31, pp. 71-82, 1984.

[8] A. L. Marshak, "The effect of the hot spot on the transport equation in plant canopies," J. Quant. Spectrosc. Radiat. Transfer, vol. L.2, pp. 615-630, 1989.

[9] R. B. Myneni, G. Asrar, and S. A. W. Gerstl, "Radiative transfer in threedimensional leaf canopies," Transport Theory and Statistical Physics, vol. 19, pp. 205-250, 1990.

[10] J.V. Dave, "Extensive datasets of the diffuse radiation in realistic atmospheric models with aerosols and common absorbing gases," Soiar Energy, vol. 21, pp. 361-369, 1978.

[11] S.P. Ahmad, E. M. Middleton, and D.W. Deering, "Computation of diffuse sky irradiances from multidirectional radiance measurements," Remote Sens. Environ., vol. 21, pp. 185-200, 1987.

[12] J.A. Weinman and P. J. Guetter, "Penetration of solar irradiances through the atmosphere and plant canopies," J. Appl. Meteorol., vol. 11 pp. 136-140, 1972.

[13] S. A. W. Gerstl and A. A. Zardecki, "Coupled atmosphere/canopy moilel for remote sensing of plant reflectance features," Appl. Opt., vol. 24, pp. $94-103,1985$.

[14] S. A. W. Gerstl and A.C. Simmer, "Radiation physics and modeling for off-nadir satellite sensing of non-lambertian surfaces," Remote Sens. Environ., vol. 20, pp. 1-29, 1985.

[15] R. B. Myneni, G. Asrar, and E. T. Kanemasu, "Light scattering in plant canopies: the method of successive orders of scattering approximation (SOSA)," Agric. For. Meteorol., vol. 39, pp. 1-12, 1987.

[16] S. A. W. Gerstl and A. A. Zardecki, "Discrete-ordinates finite-element method for atmospheric radiative transfer and remote sensing," Appl. Opt., vol. 24, pp. 81-93, 1985.

[17] R. B. Myneni, V.P. Gutschick, J. K. Shultis, G. Asrar, and E. T. Kanemasu, "Photon transport in vegetation canopies with anisotropic scatlering, part IV: Discrete-ordinates finite difference exact kernel technique for photon transport in slab geometry for the two-angle problem," Agric For. Meteorol., vol. 42, pp. 101-120, 1988.

[18] K. Cooper, J. A. Smith, and D. Pitts, "Reflectance of a vegetative canopy using the adding method," Apl. Opt., vol. 21, pp. 4112-4118, 1982.

[19] B. D. Ganopol, "The determination of the reflected intensity for a single leaf angle canopy via Chandrasekhar's method," Transport Theory and Statistical Physics, vol. 19, pp. 251-271, 1990.

[20] T. Lee and Y.J. Kaufman, "The effect of non-Lambertian surface on remote sensing of surface reflectance and vegetation index," IEEE Trans. Geosci. Remote Sensing, vol. 24, pp. 699-708, 1986.

[21] J. V. Dave, "Intensity and polarization of the radiation emerging for a plane-parallel atmosphere containing monodispersed aerosols," Appl. Opt., vol. 9, pp. 2673-2684, 1970.

[22] B. M. Herman, W. Asous, and S. R. Browning, "A semi-analytic technique to integrate the radiative transfer equation over optical depth," $J$. Atmos. Sci., vol. 37, pp. $1828-1838,1980$.

[23] S. Liang and A.H. Strahler, "An analytic canopy BRDF model anct its inversion," IEEE Trans. Geosci. Remote Sensing, in press, 1993.

[24] B.W. Herman and S. R. Browning, "A numerical solution to the equation of radiative transfer," J. Atmos. Sci., vol. 22, pp. 559-\$66, 1965.

[25] D. J. Diner and J.V. Martonchik, "Atmospheric transfer of radiation above an inhomegenous non-Lambertian reflective ground, I: Theory," J. Quant. Spectrosc. Radiat. Transfer, vol. 31, pp. 97-125, 1984.

[26] D. J. Diner and J.V. Martonchik, "Atmospheric transfer of radiation above an inhomogenous non-lambertian reflective ground, II: computational considerations and results," J. Quant. Spectrosc. Radiat. Tran:fer, vol. 32, pp. 279-304, 1984

[27] K. Stamnes, S. C. Tsay, W. Wiscombe, and K. Jayaweera, "Numerically stable algorithm for discrete-ordinate-method radiative transf $\mathrm{r}$ in multiple scattering and emitting layered media," Appl. Opt., vol. 27, pp. 2502-2509, 1988.

[28] J.P. Odom, "Neutron transport with highly anisotropic scattering," Ph.D. dissertation, Kansas State University, 1975.

[29] J. K. Ross and A. L. Marshak, "Calculation of canopy bidirectional 
reflectance using the Monte Carlo method," Remote Sens. Environ., vol. 24, pp. 213-225, 1988.

[30] C. Elterman, "Vertical attenuation model with height surface meteorological ranges 2 to 13 kilometers," Rep. No. AFCRL-70-0200, Air Force, Cambridge Research Labs., Bedford, MA, 1970.

[31] K.J. Ranson, L.L. Biehl, and M.E. Bauer, "Variation in spectral response of soybean with respect to illumination, view and canopy geometry," Int. J. Remote Sens., vol. 6, pp. 1827-1842, 1985.

[32] K. J. Ranson, L.L. Biehl, and C.S.T. Daughtry, Soybean Canopy Reflectance Modeling Data Sets, LARS Tech. Rep. 071584, 1984.

[33] M. M. Verstraete, B. Pinty, and R.E. Dickinson, "A physical model of the bidirectional reflectance of vegetation canopies, 1. Theory," $J$. Geophys. Res., vol. 95, pp. 11, pp. 755-11, 765, 1990.

[34] G.W. Suits, "The calculation of the directional reflectance of a vegetative canopy," Remote Sens. Environ., vol. 2, pp. 117-125, 1972.

[35] J.A Smith and R.E. Oliver, "Effects of changing canopy directional reflectance on feature selection," Appl. Opt., vol. 13, pp. 1599-1604, 1974.

[36] W. Verhoef, "Light scattering by leaf layers with application to canopy reflectance modeling: the SAIL model," Remote Sens. Environ., vol. 16, pp. $125-141,1984$.

[37] M. M. Verstraete, "Radiative transfer in plant canopies: Transmission of direct solar radiation and the role of leaf orientation," J. Geophys. Res., vol. 92, pp. 10985-10995, 1987.

[38] G.S. Campbell, "Extinction coefficients for radiation in plant canopies calculated using an ellipsoidal inclination angle distribution," Agric. For. Meteorol., vol. 36, pp. 317-321, 1984.

[39] N. S. Goel and D. E. Strebel, "Simple beta distribution representation of leaf orientation in vegetation canopies," Agron. J., vol. 76, pp. 800-803, 1984.

[40] N.J.J. Bunnik, The Multispectral Reflectance of Shortwave Radiation by Agricultural Crops in Relation with Their Morphological and OP tical Properties. Wageningen, the Netherlands: Mededelingen Landbouwhogeschool, 1978.

[41] C. T. de Wit, "Photosynthesis of leaf canopies," Agric. Res. Rep. No. 663, Pudoc, Wageningen, The Netherlands, 1965.

[42] C. G. Bohren, "Multiple scattering of light and source of its observable consequences," Am. J. Phys., vol. 55, pp. 524-533, 1987.

[43] J. Otterman and T.W. Brakke, "Multiple vs. single scattering: assessment of magnitudes," Appl. Opt., vol. 30, pp. 1562-1566, 1991.

[44] J. Lenoble, Radiative Transfer in Scattering and Absorbing Atmospheres: Standard Computational Procedures. Hampton, VA: A. Deepak Publ., 1985.

[45] T. Nilson and A. Kuusk, "A reflectance model for the homogeneous plant canopy and its inversion," Remote Sens. Environ., vol. 27, pp. 157-167, 1989.

[46] D. H. Card, "A simplified derivation of leaf normal spherical coordinates," IEEE Trans. Geosci. Remote Sensing, vol. 25, pp. 884-887, 1987.
[47] V.C. Vanderbilt and L. Grant, "Plant canopy specular reflectance model," IEEE Trans. Geosci. Remote Sensing, vol. 23, pp. 722-730, 1985.

[48] D. L. B. Jupp and A.H. Strahler, "A hotspot model for leaf canopies," Remote Sens. Environ., vol. 38, pp. 193-210, 1991.

[49] L. Esposito, "Extensions to the classical calculation of the effect of mutual shadowing in diffuse reflection," Icarus, vol. 39, pp. 69-80, 1979.

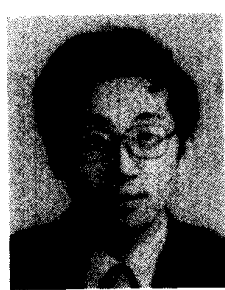

Shunlin Liang received the B.S. degree in computer cartography, the M.S. degree in remote sensing \& geographic information system from Nanjing University, People's Republic of China, and the Ph.D. degree in remote sensing from Boston University, MA.

From 1986 through 1989, he was an assistant professor and lecturer in Nanjing University. He served as a research assistant in Boston University from $1989-1992$. He is currently a postdoctoral fellow at Center for Remote Sensing and Department of Geography, Boston University. He has published papers in marine remote sensing, image processing and computer cartography. His present research interests focus on land surface-atmosphere radiative transfer modeling, and inversion from multiangle remotely sensed data.

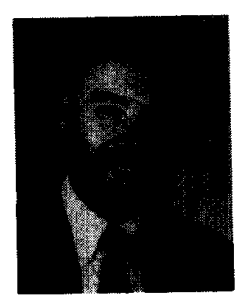

Alan H. Strahler (M'86) received the B.A. and the $\mathrm{Ph} . \mathrm{D}$. degrees in geography from The Johns Hopkins University in 1964 and 1969, respectively. $\mathrm{He}$ is currently Professor of Geography and $\mathrm{Re}$ searcher in the Center for Remote Sensing, Boston University, Boston. He has held prior academic positions at Hunter College of the City University of New York, at the University of California, Santa Barbara, and at the University of Virginia. Originally trained as a biogeographer, he has been actively involved in remote sensing research since 1978. He has been a Principal Investigator on numerous NASA contracts and grants, and is currently a member of the Science Team for the EOS MODIS

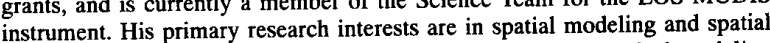
statistics as they apply to remote sensing, and in geometric-optical modeling of remotely-sensed scenes. He is particularly interested in remote sensing of forests and the inference of vegetation canopy parameters from digital images through invertible models. 